www.jmscr.igmpublication.org

Index Copernicus Value: 79.54

ISSN (e)-2347-176x ISSN (p) 2455-0450

crossrefDOI: https://dx.doi.org/10.18535/jmscr/v7i3.78

Journal Of Medical Science And Clinical Research

IGM Publication

An official Publication of IGM Publication

\title{
A Study of Cardiopulmonary Manifestations in Patients with Rickettsial Fever at a Tertiary Care Centre
}

\begin{abstract}
Authors
Dr Suman G $\mathbf{R}^{1}$, Dr Muralidhar $\mathbf{H}^{2 *}$, Dr Dharanidhar Reddy ${ }^{3}$

${ }^{1}$ Assistant Professor, Department of General Medicine, S.S. Institute of Medical Sciences \& Research

Centre, Davangere, Karnataka, India

${ }^{2}$ Post Graduate, Department of General Medicine, S.S. Institute of Medical Sciences \& Research Centre, Davangere, Karnataka, India

${ }^{3}$ Post Graduate, Department of General Medicine, S.S. Institute of Medical Sciences \& Research Centre,

Davangere, Karnataka, India

*Corresponding Author

Dr Muralidhar.H

${ }^{2}$ Post Graduate, Department of General Medicine, S.S. Institute of Medical Sciences \& Research Centre,

Davangere, Karnataka, India

Abstract

Background and Objectives: Rickettsial diseases are considered some of the most important emerging diseases which are increasingly being recognized in India. The clinical spectrums of rickettsial diseases are broad, with most infections being of mild-to-moderate severity. Cardiopulmonary manifestations are quite common as the severity of rickettsial disease increases. This study aims at studying the cardiopulmonary manifestations in patients with rickettsial fever. Prompt antibiotic therapy, even based on suspicion, shortens the course of the disease lowers the risk of complications and in turn reduces morbidity and mortality due to rickettsial diseases.

Materials and Methods: The present study is a cross sectional observational study. A thorough history taking and clinical examination was conducted on patients admitted with acute febrile illness. The necessary investigations for these patients were sent, the reports of which were collected later on. The cases which showed Weil-Felix test positivity with 1:80 and above titres were analyzed.

Results: Weil-Felix positive patients with 1:80 titers or above had fever (100\%), chills/rigors (75.6\%), myalgia (67.9\%),dyspnoea (32.1\%) and cough (46.2\%).Considering signs, $17.9 \%$ had pallor, $48.7 \%$ had tachycardia, $17.9 \%$ had bradycardia and $51.3 \%$ had respiratory signs during examination. 8 patients who had ECG changes had sinus tachycardia (3) and sinus bradycardia (5). Of the 78 patients 19 patients had pleural effusion and 21 patients had ARDS.

Intepretation and Conclusion: Clinical manifestations like fever, myalgia and cough were quite common in rickettsial fever patients. More than half the patients presented with respiratory signs like tachypnoea, creptations, rhonchi and reduced breath sounds. 21 patients demonstrated features of ARDS on chest radiography. This study emphasizes the fact that a diagnosis of rickettsial fever should be suspected, when a patient presents with fever and respiratory signs/symptoms along with cardiovascular manifestations, as early detection of the disease could reduce morbidity \& mortality.

Keywords: Rickettsial fever, typhus group, spotted group, scrub group.
\end{abstract}




\section{Introduction}

Rickettsiae comprise a group of microorganisms that phylogenetically occupy a position between bacteria and viruses. Rickettsial diseases are considered some of the most important emerging diseases which are increasingly being recognized in India. These infections have been reported from various states and union territories like Maharashtra, Delhi, Karnataka, West Bengal, Pondicherry, Kerala, Tamil Nadu, Himachal Pradesh, Jammu and Kashmir, Rajasthan, Meghalaya, Manipur, Goa and Uttarakhand. ${ }^{1}$

The lack of proper clinical diagnostic techniques in low-income settings such as India contributes to a delay in starting treatment. Diagnosis and surveillance of this disease can be challenging, particularly in the absence of advanced laboratory diagnostic techniques. Although rickettsiae can be isolated from or detected in clinical specimens, serological tests still remain the main tool for the diagnosis. ${ }^{2}$ Even though the specific gold standard tests for diagnosing rickettsial infections are the immunofluorescence antibody (IFA) and the indirect imunoperoxidase (IP) test; these are expensive and not easily available in all parts of India. As a result, weil felix becomes a useful, less expensive, easily available test in establishing presumptive diagnosis in cases of rickettsial fever. $^{3}$

This study helps to study the cardiopulmonary complications of rickettsial diseases in patients admitted to a tertiary care hospital.

\section{Objectives of the Study}

1. To study the cardiovascular and pulmonary manifestations in patients with rickettsial fever.

2. To aid in the early diagnosis and treatment of rickettsial fever.

\section{Methodology}

The present study on "A Study of Cardiopulmonary Manifestations In Patients With Rickettsial Fever At A Tertiary Care Centre"' is an observational study.

\section{Inclusion Criteria}

Inpatients above 18 years with fever and positive Weil Felix test with titres 1:80 or above.

\section{Exclusion Criteria}

1. Patients older than 80 years and less than 18 years.

2. Patients diagnosed with other causes of acute febrile illness, e.g. dengue, malaria, leptospirosis, enteric fever, chikungunya.

3. Immunocompromised and patients suffering from chronic diseases.

4. Underlying cardiopulmonary diseases.

\section{Methodology}

A thorough history taking and clinical examination was conducted on patients admitted to the department of general medicine with fever. The necessary investigations for these patients were sent, the reports collected. The cases which showed weil felix test positivity with 1:80 titres or above were analyzed along with the clinical features to gain a complete perspective of rickettsial fever follow-up.

\section{Investigations}

- History, Examination and Informed consent were taken.

- A set of routine blood investigations including Complete Blood Count and ABG.

- Weil- Felix test - The test was performed by the slide method using the commercially available antigens (OX19, OX2 and OXK).

- Pripheral smear/card test for malaria, Serological Profile for Dengue, Leptospira IgM ELISA, WIDAL/IgM for thyphoid, Chikungunya IgM for Chikungunya were done and tabulated and interpreted in this study.

- Other radiological investigations including chest xray and ECHO were also done and interpreted. 


\section{Results}

\section{1) Symptoms}

Table 1: Presenting Symptoms

\begin{tabular}{|l|c|c|}
\hline Symptoms & Frequency & Percent \\
\hline Fever & 78 & 100.0 \\
\hline Chills/Rigors & 59 & 75.6 \\
\hline Myalgia & 53 & 67.9 \\
\hline Dyspnoea & 25 & 32.1 \\
\hline Cough & 36 & 46.2 \\
\hline Chest Pain & 8 & 10.2 \\
\hline
\end{tabular}

Various signs and symptoms of cardiovascular and respiratory system in rickettsial fever is tabulated below. Out of 78 patients 25 had dyspnoea accounting for $32.1 \%$ and 36 had cough accounting for $46.2 \%$. Chest pain was observed in 8 patients.

\section{2) Signs}

We observed pallor in 14 patients, tachycardia in 38 patients, bradycardia in 14 patients and hypotension in 21 patients at the time of admission. 40 patients out of 78 had respiratory signs.

Table 2: Presenting Signs

\begin{tabular}{|l|c|c|}
\hline Signs & Frequency & Percent \\
\hline Pallor & 14 & 17.9 \\
\hline Tachycardia & 38 & 48.7 \\
\hline Bradycardia & 14 & 17.9 \\
\hline Hypotension & 21 & 26.9 \\
\hline Respiratory Signs & 40 & 51.3 \\
\hline
\end{tabular}

\section{3) Chest X-Ray Findings}

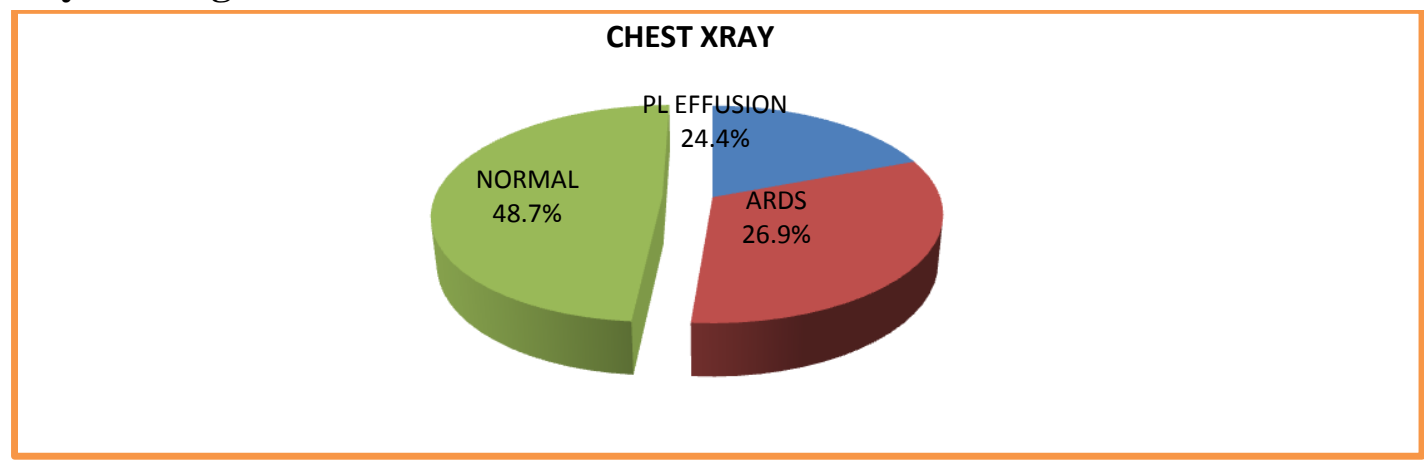

Graph 1: Chest X-Ray

Below table and graph demonstates the chest $\mathrm{x}$ ray findings in rickettsial fever. 21 patients out of 78 had features of ARDS and 19 patients had pleural effusion.

Table 3: Chest Xray Findings

\begin{tabular}{|l|c|c|}
\hline Chest Xray & Frequency & Percent \\
\hline Ards & 21 & 26.9 \\
\hline Pleural Effusion & 19 & 24.4 \\
\hline Normal & 38 & 48.7 \\
\hline Total & 78 & 100.0 \\
\hline
\end{tabular}

\section{4) ARDS}

Using ABG, ARDS was divided into mild(6), moderate(11) and severe ARDS(4).

Table 4: Severity of ARDS

\begin{tabular}{|l|c|c|}
\hline ARDS & Frequency & Percent \\
\hline Mild & 6 & 28.5 \\
\hline Moderate & 11 & 52.4 \\
\hline Severe & 4 & 19.1 \\
\hline Total & 21 & 100.0 \\
\hline
\end{tabular}

\section{5) ECG Findings}

Table 5: ECG Findings

\begin{tabular}{|l|c|c|}
\hline ECG & Frequency & Percentage \\
\hline Sinus Tachycardia & 38 & 17.9 \\
\hline Sinus Bradycardia & 14 & 48.7 \\
\hline Normal & 26 & 33.3 \\
\hline Total & 78 & 100.0 \\
\hline
\end{tabular}

We observed that out of 78 patients admitted with Rickettsial fever, 38 patients had sinus tachycardia as the ECG finding and 14 had sinus bradycardia for further echocardiography evaluation was done. 


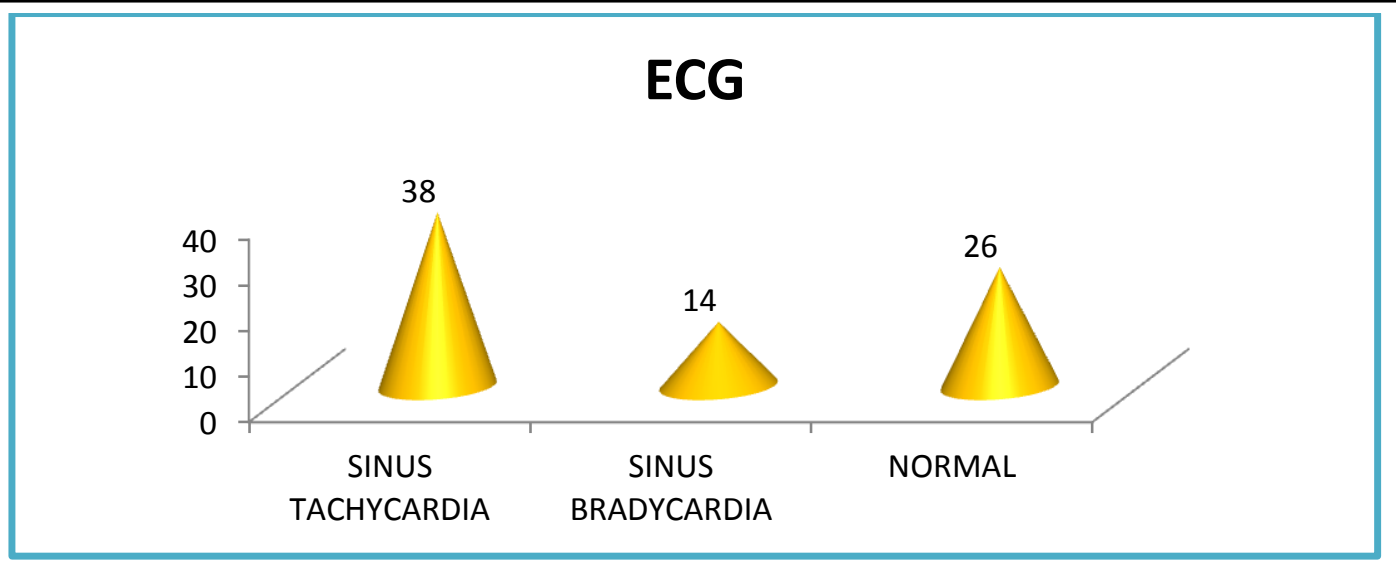

Graph 2: ECG Findings

\section{6) Echo Findings}

Table 6: Echo Findings

\begin{tabular}{|l|c|c|}
\hline Echo & Frequency & Percentage \\
\hline Global Hypokinesia & 14 & 17.9 \\
\hline Normal & 64 & 82.1 \\
\hline Total & 78 & 100.0 \\
\hline
\end{tabular}

14 patients had sinus bradycardia in which ECHO shows global hypokinesia. Rest was normal.

\section{7) Symptoms with Antigenic Variants}

In the following table we observed that 23 patients out of 59 who presented with chills were OX $19+$ OX $2+$ OX K positive patients. 10 patients had dyspnoea as presenting symptom in OX19+ OX $\mathrm{K}+\mathrm{OX} 2$ positive patients. Out of 36 patients who presented with cough, 13 were observed in OX K + OX $19+$ OX 2 positive patients. Chest pain was observed in 8 patients with maximum seen in OX K + OX $19+$ OX 2 positive patients.

Table 7: Antigenic Variants with Symptoms

\begin{tabular}{|l|c|c|c|c|c|}
\hline ANTIGEN & N & CHILLS & CHEST PAIN & COUGH & DYSPNOEA \\
\hline OX 2 & 13 & 9 & 1 & 3 & 2 \\
\hline OX 19 & 1 & 1 & 0 & 0 & 0 \\
\hline OX K & 10 & 8 & 2 & 5 & 4 \\
\hline OX 2 + OX 19 & 9 & 6 & 1 & 5 & 2 \\
\hline OX 19 + OX K & 5 & 3 & 1 & 2 & 6 \\
\hline OX 2 + OX K & 14 & 9 & 0 & 8 & 10 \\
\hline OX 19+ OX 2 + OX K & 26 & 23 & 3 & 13 & $\mathbf{2 5}$ \\
\hline Total & $\mathbf{7 8}$ & $\mathbf{5 9}$ & $\mathbf{8}$ & $\mathbf{3 6}$ & \\
\hline
\end{tabular}

\section{8) Antigenic Variants with Signs}

In table below, pallor was observed in 14 patients of which maximum (4) were seen in OX 2 positive patients. Maximum tachycardia was observed in OX $2+$ OX K positive patients (10)

Table 8: Antigenic Variants with Signs

\begin{tabular}{|l|c|c|c|c|c|}
\hline Antigen & N & Pallor & Tachycardia & Bradycardia & Hypotension \\
\hline OX 2 & 13 & 4 & 3 & 3 & 4 \\
\hline OX 19 & 1 & 1 & 1 & 0 & 0 \\
\hline OX K & 10 & 1 & 6 & 1 & 3 \\
\hline OX 2 + OX 19 & 9 & 2 & 6 & 1 & 0 \\
\hline OX 19 + OX K & 5 & 2 & 1 & 1 & 3 \\
\hline OX 2 + OX K & 14 & 1 & 10 & 6 & 9 \\
\hline OX 19 + OX 2 + OX K & 26 & 3 & 11 & $\mathbf{1 4}$ & $\mathbf{2 1}$ \\
\hline Total & $\mathbf{7 8}$ & $\mathbf{1 4}$ & $\mathbf{3 8}$ & & \\
\hline
\end{tabular}

and OX $19+$ OX K + OX 2 positive patients (11). 6 out of 14 patients who had bradycardia were all 3 antigens positive. Hypotension was also observed maximally (9) in all 3 antigens positive patient. 


\section{Discussion}

Rickettsial infections are distributed throughout the world and are re-emerging in the Indian subcontinent, especially among children. Rickettsial disease has been reported from various parts of India, such as Tamil Nadu, Karnataka, Kerala, Maharashtra, and some parts of northern India ${ }^{4}$. The infection has established itself as an endemic disease in Southeast Asia including Thailand, China, and Taiwan ${ }^{5}$. Multiple factors contribute to the gross under-diagnosis of rickettsial infections; these include the relatively non-specific disease presentation, low index of suspicion, and lack of awareness about its reemergence. The clinical manifestations including signs and symptoms and routine haematological manifestations forms an important tool to predict diagnosis and for the management of the patients. In our study all patients had fever. More than half $(64.1 \%)$ presented with fever of 7-14 days duration. 14(17.9\%) each patients presented to us with fever for more than 2 weeks and less than 1 week duration. Fever is due to the release of endogenous and exogenous pyrogens. Endogenous pyrogens include cytokines, interleukins $1 \& 6$, TNF alpha and interferons. Endotoxins released directly affect thermoregulation in the hypothalamus as well as stimulate endogenous pyrogen release. ${ }^{6}$ More than half the patients with fever also had chills/rigors and myalgia.

A large number of patients in our study presented with symptoms pertaining to respiratory system. Respiratory tract involvement is a common manifestation of scrub typhus and clinicians need to differentiate it from community-acquired pneumonia caused by the usual organisms like streptococcus species. ${ }^{7}$ Half of the patients had signs of consolidation on clinical examination. Chest radiograph abnormalities in the form of reticulonodular opacities, air space consolidation, peribronchial infiltration, pulmonary congestion, pulmonary oedema, acute respiratory distress syndrome (ARDS) and pleural effusion were known to occur in scrub typhus. ${ }^{8,9}$ Chest X-ray abnormalities seen in our study included pleural effusion and features of ARDS seen in approximately half the patients. Patients with $\mathrm{x}$ ray features of ARDS were classified into mild, moderate and severe based on the $\mathrm{PaO} 2 / \mathrm{FiO} 2$ levels and the majority of patients came under moderate ARDS.

Table 9: Severity of ARDS ${ }^{10}$

\begin{tabular}{|l|l|}
\hline ARDS Severity & Pa02/Fi02 \\
\hline Mild & $200-300$ \\
\hline Moderate & $100-200$ \\
\hline Severe & $<100$ \\
\hline
\end{tabular}

The existence of myocarditis in scrub typhus is easily ignored, because the symptoms of myocardial involvement are usually subclinical and sometimes may lead to heart failure. ${ }^{11}$ Cardiac conduction abnormalities in the form of bradycardia were seen on electrocardiogram in 14 patients and tachycardia in 38 patients. Echo in patients with bradycardia showed global hypokinesia with reduced ejection fraction which was suggestive of myocardities. In the background of sepsis, transient cardiac dysfunction can occur due to sepsis induced cardiomyopathy.

In our study Weil felix was used as the diagnostic test with 1:80 being the cut-off. Weil-Felix test has been used for the rapid diagnosis of acute cases of infection in areas with a high prevalence. $^{12}$ The Weil-Felix (WF) test is based on the detection of antibodies to various Proteus species which contain antigens with cross-reacting epitopes to antigens from members of the genus Rickettsia. ${ }^{13}$ Mixed antigenic infection including all 3 antigenic variants patients presented with maximum number of patients with fever with chills and myalgia. Similarly, maximum patients presenting with dyspnoea and cough also belonged to mixed infection with all the antigenic variants. Scrub typhus group (positive OX K) was seen in 10 patients who presented fever, chills, myalgia, cough and breathlessness in almost half of its cases. Amano et al12 observed that of the 
sera which were positive to Rickettsia tsutsugamushi by indirect immunoperoxidase test, approximately $80 \%$ sera were positive to a Proteus OXK antigen by WF test at 10 or more days after the onset of fever. ${ }^{14}$ Though Weil-Felix agglutination test is not a very sensitive test but when positive, it is rather specific test. ${ }^{15}$

All over the country the proportion of rickettsial fever in cohorts analysing febrile illness is found to have increased significantly over the past two decades. It is believed that increasing use of these antibiotics for treatment of febrile illnesses in the community during recent times may be contributing to unmasking of rickettsial fever as rickettsiae are inherently resistant to them. ${ }^{16}$

The optimal duration of treatment has not been established, but current recommendation suggests at least 3-7 days for life threatening cases to a maximum of 15 days for severe or complicated disease. Rapid resolution of fever following doxycycline is so

characteristic that it can be used as a therapeutic test. ${ }^{17}$ The treatment of choice for scrub typhus infection is doxycycline $100 \mathrm{mg}$ per dose administered twice daily (orally or intravenously) for adults or $2.2 \mathrm{mg}$ per $\mathrm{Kg}$ for children less than $45.5 \mathrm{Kg} .{ }^{18}$ Alternatively chloramphenicol $(500 \mathrm{mg}$ 4 times a day orally for 7 days in adults or $150 \mathrm{mg}$ per kg per day for 5 days in children) in endemic areas has been proven effective in treating scrub typhus and preventing relapse. ${ }^{19}$ Rifampicin or azithromycin are effective in doxycycline resistant strains of scrub typhus. $^{20}$

The main cause of death in most of Indian studies is ARDS and less so in Taiwan. ${ }^{21,22}$ The low mortality in Taiwan may be due to the endemicity of the disease and familiarity of the disease among the clinicians. The deaths in our series are possibly due to delayed diagnosis, late presentation and multiorgan dysfunction.

\section{Conclusion}

Rickettsial fever can affect all the age groups with middle age being most commonly affected.
The salient clinical features include fever with rashes with presence of eschar being the most diagnostic. It can affect all systems with symptoms of respiratory tract being cough and breathlessness and $\mathrm{x}$-ray features include pleural effusion and ARDS. Those presenting with myocarditis have a poorer prognosis. With regard to antigenic variation using cut off value of $1: 80$ in weil-felix test majority of patients had mixed infection with OX K, OX19 and OX 2 positivity.

\section{Summary}

1) The present study on "a study of cardiopulmonary manifestations in patients with rickettsial fever at a tertiary care centre" is an observational study.

2) All blood samples which were positive for rickettsial fever on Weil-Felix test were included in the study.

3) Antigen suspension of Proteus OX 19 antigen reacts strongly with the sera of patients with typhus group Rickettsia and Rocky Mountain spotted fever, Proteus OX 2 with the sera of patients with spotted fever infections, while the Proteus OX K with the sera of patients infected with scrub typhus

4) Fever was the most common presentation in all the 78 patients $(100 \%)$.

5) Respiratory symptoms like cough were present in 36 patients and dyspnoea in 25 patients. $51.3 \%$ patients had respiratory signs. X-ray abnormality in the form of pleural effusion was seen in 19 patients and 21 patients showed features of ARDS.

6) $48.7 \%$ patients had sinus bradycardia and $17.9 \%$ patients had tachycardia. 14 out of 78 patients had global hypokinesia on Echo.

\section{Bibliography}

1. Mahajan SK, Rolain JM, Sankhyan N, Kaushal RK, Raoult D. Pediatric Scrub Typhus in Indian Himalayas. Indian Journal of Pediatrics 2008;75(9):947-9.

2. Kumar K, Jain S, Kumar A. Outbreak Indian tick typhus amongst residents of 
Deol village, District, Kangra, Himachal Pradesh (INDIA). Int J Med Public Health 2011;1:67-71.

3. Tilak R, Kunwar R, Wankhade UB, Tilak VW. Emergence of Schoengastiella ligula as the vector of scrub typhus outbreak in Darjeeling: Has Leptotrombidium deliense been replaced? Indian J Public Health 2011;55:92-9.

4. Dass R, Deka NM, Duwarah SG, Barman H, Hoque R, Mili D, Barthakur D (2011) Characteristics of pediatric scrub typhus during an outbreak in the North Eastern region of India: peculiarities in clinical presentation, laboratory findings and complications. Indian J Pediatr 78: 13651370.

5. Chanta C, Chanta S (2005) Clinical study of 20 children with scrub typhus at Chiang Rai Regional Hospital. J Med Assoc Thai 88: 1867-1872.

6. Viswanathan $\mathrm{S}$, Muthu $\mathrm{V}$, Iqbal $\mathrm{N}$, Remalayam B, George T. Scrub Typhus Meningitis in South India- A Retrospective Study. PLoS ONE 2013;8(6):1-8.

7. Subbalaxmi MVS, Madisetty MK, Prasad AK, Teja VD, Swaroopa K, Chandra N. Outbreak of Scrub Typhus in Andhra Pradesh - Experience at a Tertiary Care Hospital. Journal of the association of physicians of India 2014;62;490-6.

8. Wu K-M, Wu Z-W, Peng G-Q, et al. Scrub typhus: radiologic pulmonary findings, clinical manifestations and serious complications in scrub typhus: experiences from a teaching hospital in Eastern Taiwan. Intl J Gerontol 2009;3:223-32.

9. Charoensak A, Chawalparit O, Suttinont C, et al. Scrub Typhus: chest radiographic and clinical findings in 130 Thai Patients. J Med Assoc Thai 2006;89:600-07.

10. Kasper, Dennis L, Fauci et al. Harrison's Principles of Internal Medicine. 19th edition. New York: McGraw Hill Education, 2015.
11. Tsay RW, Chang FY. Serious complications in scrub typhus. J Microbiol Immunol Infect 1998;31:240-4.

12. La Scola B, Raoult D. Laboratory Diagnosis of Rickettsioses: Current Approaches to Diagnosis of Old and New Rickettsial Diseases. J Clin Microbiol 1997;35:2715-27.

13. Amano K, Suzuki N, Fujita M, et al. Serological reactivity of sera from scrub typhus patients against Weil-Felix test antigens Microbiol Immunol 1993;37:92733.

14. Amano K, Suzuki N, Hatakeyama H, et al. The reactivity between rickettsiae and Weil-Felix test antigens against sera of rickettsial disease patients. Acta Virol 1992; 36:67-72.

15. Saah AJ Orientia tsutsugamushi (Scrub Typhus) In Mandell GL, Bennet JE, Doalin R, Edr. Principles and Practice of Infectious Diseases. Philadelphia: Churchill Livingstone; 2000:2056-7.

16. Chandy S, Thomas K, Mathai E, Antonisamy B, Holloway K, al e. Patterns of antibiotic use in the community and challenges of antibiotic surveillance in a lower-middleincome country setting: a repeated cross-sectional study in Vellore, South India. J Antimicrob Chemother. 2013; 68: 229 - 236.

17. Kim D, Kang D, Kim J, Chung J, Kim H, et al. Acute renal failure due to acute tubular necrosis caused by direct invasion of Orientia tsutsugamushi. Journal of clinical microbiology. 2008; 46: 15481550.

18. Nachega JB, Bottieau E, Zech F et al. Travel-acquired scrub typhus: emphasis on the differential diagnosis, treatment, and prevention strategies. $J$ Travel Med 2007;14:352-5.

19. Isaac R, Varghese GM, Mathai E, et al. Scrub Typhus: Prevalence and Diagnostic 
Issues in Rural Southern India. Clin Infect Dis 2004;39:1395-6.

20. H Boorugu, M Dinaker, ND Roy, et al. Reporting a case of Scrub Typhus from Andhra Pradesh. J Assoc Physicians India 2010;58:520.

21. Vivekanandan M, Mani A, Priya YS, et al. Outbreak of scrub typhus in Pondicherry. $J$ Assoc Physicians India 2010;58:24-28.

22. Sharma P, Kakkar R, Shilpa NK, et al. Geographical distribution, effect of season and life cycle of scrub typhus. JK Science 2010;12:63-4. 